\title{
Evolution of Bounded Confidence Opinion in Social Networks
}

\author{
Hui Xie, ${ }^{1,2}$ Guangjian Li, ${ }^{1}$ Yongjie Yan, ${ }^{2}$ and Sihui Shu ${ }^{2}$ \\ ${ }^{1}$ Department of Information Management, Peking University, Beijing 100871, China \\ ${ }^{2}$ School of Mathematics and Computer Science, Jiangxi Science \& Technology Normal University, Nanchang 330038, China \\ Correspondence should be addressed to Guangjian Li; ligj@pku.edu.cn
}

Received 22 February 2017; Accepted 15 March 2017; Published 3 April 2017

Academic Editor: Luisa Di Paola

Copyright (C) 2017 Hui Xie et al. This is an open access article distributed under the Creative Commons Attribution License, which permits unrestricted use, distribution, and reproduction in any medium, provided the original work is properly cited.

\begin{abstract}
We investigate opinion dynamics as a stochastic process in social networks. We introduce the stubborn agent in order to determine the impact of network structure on the emergence of consensus. Depending on the fraction of undirected long-range connections, we observe fascinatingly rich dynamical behavior and transitions from disordered to ordered states. In general, we find that the stubborn agent promotes the emergence of consensus due to the so-called "group effect" that facilitates coalescence between separated network components. Agents are also behaviorally constrained Shannon information entropy in networks. However, since agents want to evolve their opinion with Brownian motion, which may in turn impede full consensus, sufficiently frequent long-range links are in such situations crucial for the network to converge into an absorbing phase. Our experimental findings indicate that, for a large range of control parameters, our model yields stable and fluctuating polarized states.
\end{abstract}

\section{Introduction}

The study of opinion dynamics, which links social behavior with topics of statistical physics, is rapidly attracting the attention of scholars in many disciplines. Indeed, methods of statistical physics have had a significant impact on the study of social systems $[1,2]$, as well as on a number of related interdisciplinary fields of research, ranging from ecology to sociology. In recent years, fascinatingly interesting phase transitions in various models of social phenomena have been reported, and ample efforts are invested towards the study of socially inspired models, such as the voter model [3$5]$, the majority rule model $[6,7]$, the social impact model $[8,9]$, the bounded confidence model $[10-12]$, the Sznajd model $[13,14]$, and many related agent-based models $[15,16]$. Of particular relevance for the present study are models where the phenomenon of coexistent opinions, emerging from a polarized state due to additional constraints, has been reported $[17,18]$.

The process of opinion forming is most often highly predictable on the topology of the underlying interaction network $[19,20]$. Interaction structures such as the scalefree networks take into account social, natural, and economic factors much more relevantly than traditionally considered lattices and regular networks. The interest has been intensified recently by the rapid growth of online social networks (e.g., Twitter, Facebook, and Weibo), which provide the basis for a better understanding of opinion formation. In social networks, not all players have an equal number of connections. These important features need to be taken into account in opinion dynamics by using the formalism of Monte Carlo simulations and agent-based modeling.

Scale-free networks [19] are considered as proper and quite accurate representations of real-world social networks, where individuals have not only short-range, but also longrange connections. Inferring phase transitions in models with social interaction, including those describing opinion formation, is difficult as decisions are seldom made sequentially, players are not aware of the decisions of others, and they can change their opinion at any moment during the evolutionary process. In general, socially inspired agent-based models are significantly more complex than agent-based models of physical systems [21], and therefore well-mixed models described by means of a dynamical system are frequently employed, also for the study of opinion formation, although bright and very noteworthy exceptions are more and more common [22]. 
In this paper, we study opinion formation with the stubborn agents in social networks and subject to group evolution. The model is based on the modified bounded confidence model for the description of the dynamics of the contact process with long-range correlations. We also discuss thermodynamic process in opinion evolution by using Shannon information entropy [23]. In the proposed model, at each step of the simulation, an agent can change its opinion by interacting with its neighbors or its network topology once on average. In what follows, we first provide an accurate description of the model and then proceed with the presentation of the main results and the conclusions.

\section{Evolution Opinion Dynamic with Stubborn Agents}

As scale-free networks [19], social networks display a lot of heterogeneity in nodes connectivity. In order to study opinion dynamics in a large group of agents, we consider a network with $n$ agents at a discrete time step $t$. The subsequent opinion of agent $i$ is affected by its close neighbors $j$ 's, as well as the average opinion of the whole network (feedback effect). Parameters are varied in numerical simulations to study turbulent-like dynamics. Relations in the network can be spatial neighborhood, friendship, financial cooperation, group sport activities, and so forth.

As the basis of the simulation with the time-variant model, we use several different matrices to explore a sparse matrix. At time $t$, the opinion of agent $i \in(1, \ldots, n)$ with two internal parameters: an opinion $x_{i}(t)$ and an age $a_{i}(t)$.

At start time, the agents do not know each other, so that every agent puts same weight on every other agent, except himself. Each agent $i$ can be influenced by another agent $j$, if the opinions of these two agents are closer than a real number $d$, where $d$ is the value of the threshold of influence, while stubborn agents are a group of explicit entities, which are indistinguishable from normal agents. As a key concept in statistical thermodynamics and information theory, entropy has been used as a measure to characterize properties of the topology in complex networks. Let $k_{1}, k_{2}, \ldots, k_{n}$ be independent and identically distributed random variables in a finite set $K$ with $p\left(k_{i}\right)$, which denotes the probability of an agent to be in a degree of connectivity $k$ in scale-free networks. Shannon information entropy $H$ of node $i$ can be obtained by

$$
H\left(k_{i}\right)=-\sum_{j=1}^{n} p\left(k_{i j}\right) \log p\left(k_{i j}\right)
$$

where $n$ is the total number of nodes included in the network and $p\left(k_{i j}\right)$ is the proportion of $i$ 's total weight volume that points to $j$. Then the Shannon information entropy $H$ of set $K$ can be written as

$$
H(K)=\sum_{i=1}^{n} H\left(k_{i}\right)=-\sum_{i=1}^{n} \sum_{j=1}^{n} p\left(k_{i j}\right) \log p\left(k_{i j}\right) .
$$

The entropy rate of an agent depends both on the dynamical process and on the graph topology in networks.
In opinion formation, we can also use it as a measurement to quantify the diversity of opinion. Furthermore, a stubborn agent satisfies the interaction condition for all its neighbors. However, a normal agent changes his opinion in course of interaction and may be unable to fulfill the interaction condition with some of its neighbors.

At each time step $t$, an agent $x_{i}(t)$ and one of its neighbors $x_{j}(t)$ are chosen at random. Depending on the types, there are different update rules to be applied.

(a) Two normal agents meet: If $\Delta x=\left|x_{i}(t)-x_{j}(t)\right|<d$ at time $t$, opinions are adjusted according to

$$
\begin{aligned}
& x_{i}(t+1)=x_{i}(t)+\mu \cdot\left(x_{j}(t)-x_{i}(t)\right) \\
& x_{j}(t+1)=x_{j}(t)+\mu \cdot\left(x_{i}(t)-x_{j}(t)\right),
\end{aligned}
$$

where $\mu$ is a convergence rate whose values may range from 0 to 0.5 ; for $\mu=0.5$, the agents adjust their opinion to the average of both opinions.

(b) A normal and a stubborn agent meet: Without loss of generality assume $i$ is the stubborn agent, which is reluctant to change its opinion, and $j$ is the normal agent. We define the probability of convergence $p_{\text {inter }}$ for two agents $i$ and $j$ with an opinion difference $\Delta x=\left|x_{i}-x_{j}\right|$ as

$$
p_{\text {inter }}=\left[1+\exp \left(\frac{\Delta x / d-1}{s}\right)\right]^{-1},
$$

where parameter $s$ resembles a probability of stubborn in the steepness of the convergence. A small $s$ value means that probability of stubborn is steep, while a large value indicates that an opinion difference is important.

(c) Two stubborn agents meet: Nothing happens.

\section{Results in Social Networks}

The Barabási-Albert (BA) scale-free network primary goal is to reproduce the growth processes taking place in real networks, and it is based on two basic ingredients: growth and preferential attachment. We crawled and collected profiles of 100000 users on Weibo, a Twitter-like microblogging service in China, from 7th July to 10th July in 2016. The number of the corrected data is $N=5000$ with removing inactive users. The degree distribution resulting from the model is BA scale-free, with the average degree $\langle k\rangle=13$, and the power law exponents of degree distribution $\gamma=2.6$. We carry out simulations of the data as shown in Figure 1.

We run simulations to get an insight of opinion dynamics with different numbers of agents. Figure 2 suggests that the convergence to consensus usually takes place in the center. Big blocks of agents are built at about time step eight, and two small groups of agents survive. Although it looks stable, this situation will lead to slow convergence to consensus at time step eighty. It means that under value of $n=1000$, appropriate number of agents (such as 450 or 550) is a better choice in the system.

The results of opinion evolution with different numbers of agents are shown in Figure 3.

We have performed similar finite size scaling analyses for a variety of other points in the parameter space. In Figure 4, 


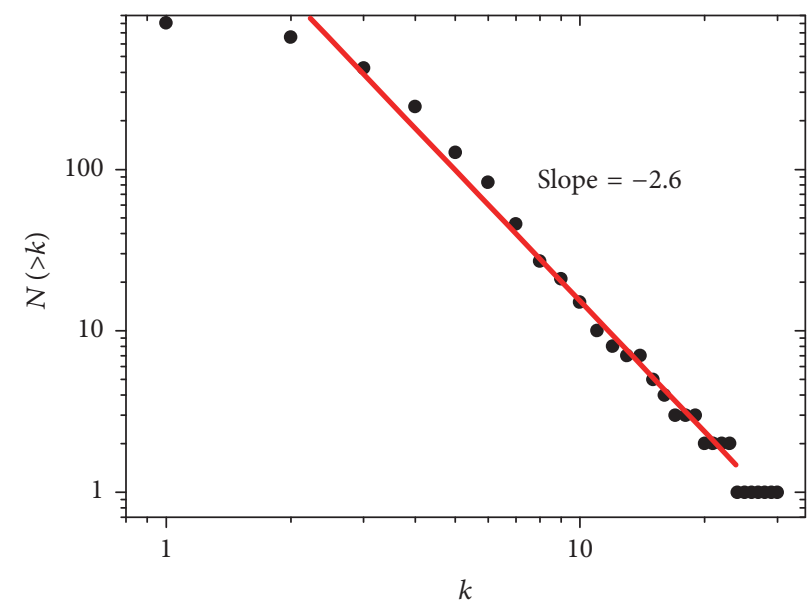

FIGURE 1: The degree follows (heavy tailed) power law distribution with slope $=-2.6$ in our data.

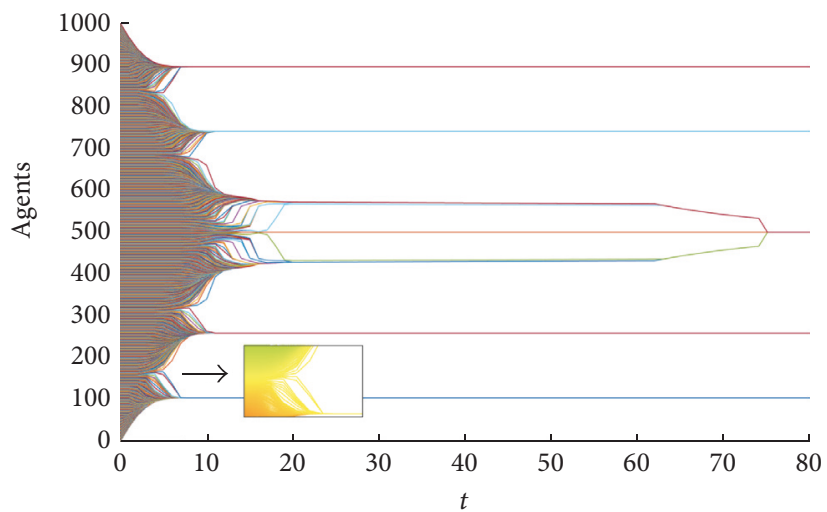

FIgURE 2: The number of agents $n$ determines the whole opinion profile in the random distribution of opinions in the network.

we show plots of $\Delta t$ steps with $N_{i} \in 1,2, \ldots, 5000$ in our model.

Further analytical treatment can be performed for the case of convergence state. An opinion group with $N_{i}$ agents at time $t$ has mean opinion $x_{i}(t)$ which evolves according to Brownian motion, if new agents with opinion $x_{i}$ appear inside its region of attraction $x_{i}(t)-d \leq x_{i} \leq x_{i}(t)+d$. In convergence state, this gives an expected distance covered by diffusion after $\Delta t$ steps of

$$
E\left(\left|x_{i}(t+\Delta t)-x_{i}(t)\right|\right)=\sqrt{\frac{2 \Delta t}{\pi} \frac{\mu d}{2 N_{i}}} .
$$

The relationship of $\Delta t$ and $E$ in the system is shown in Figure 5. The distribution of $E$ and curve of $\Delta t$ are approximate fitted, under agent's dynamic with Brownian motion.

\section{Conclusion}

In this paper, We have proposed and studied opinion formation in BA scale-free networks with the stubborn agents by performing Monte Carlo calculations and mean-field approximations. The model merges the classical opinion formation dynamics of the bounded confidence model with Brownian motion. The effect of long-range directed links between agents on the opinion formation has been systematically investigated. The phase transition behavior varies depending on how likely the players change their own opinion, that is, what their respective probability value of $p_{\text {inter }}$ is.

In the latter case, the dynamics becomes very interesting and rich, as demonstrated by the variety of ways in which consensus can be reached. Altogether, our results outline a systematic picture of opinion formation in BA scale-free networks and provide fascinating insight into the effects of group-structural dynamics on the emergence of consensus.

\section{Conflicts of Interest}

The authors declare that they have no conflicts of interest.

\section{Acknowledgments}

This work was sponsored by the National Natural Science Foundation of China (71561013), the Major Project of the National Social Science Foundation of China (15ZDB129), the Key Project of the National Social Science Foundation 


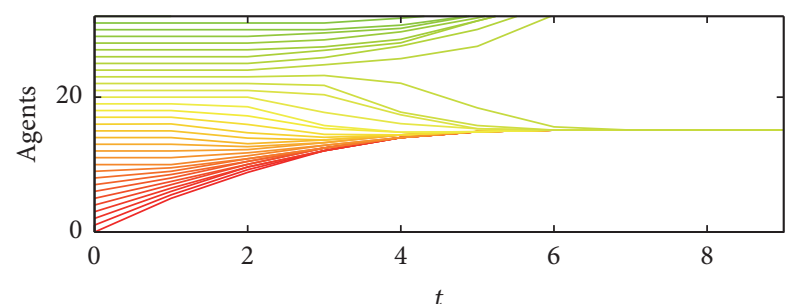

(a)

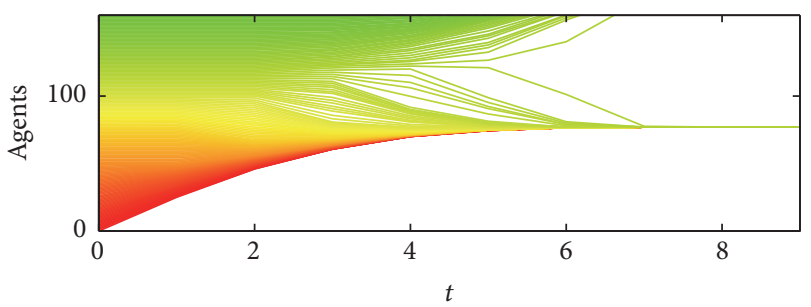

(c)

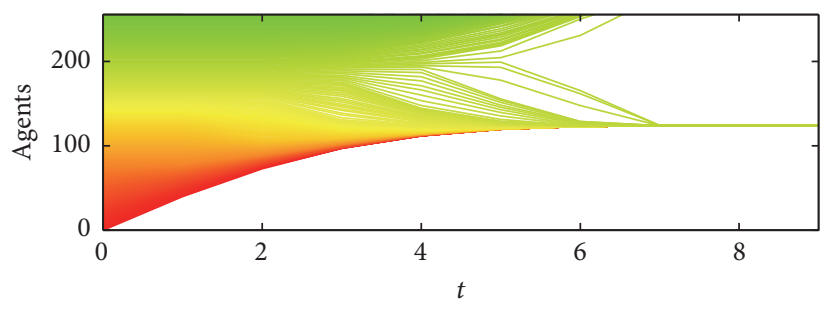

(e)

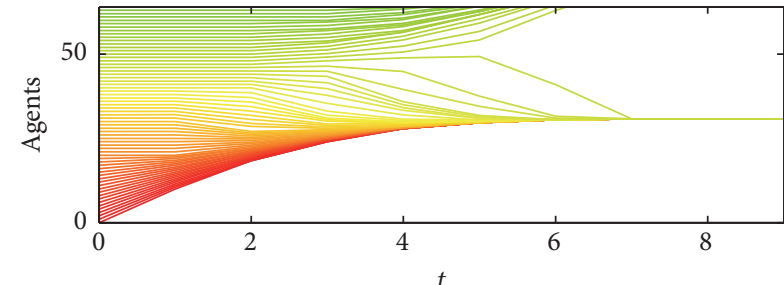

(b)

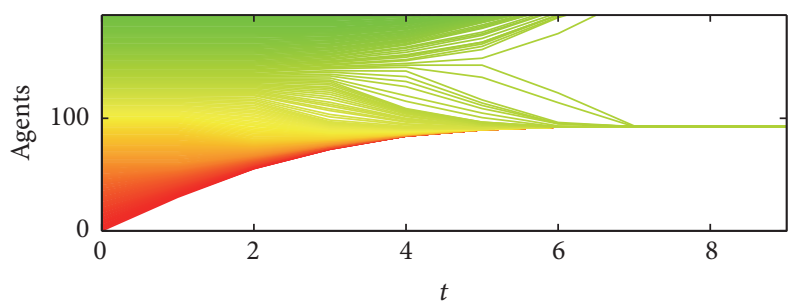

(d)

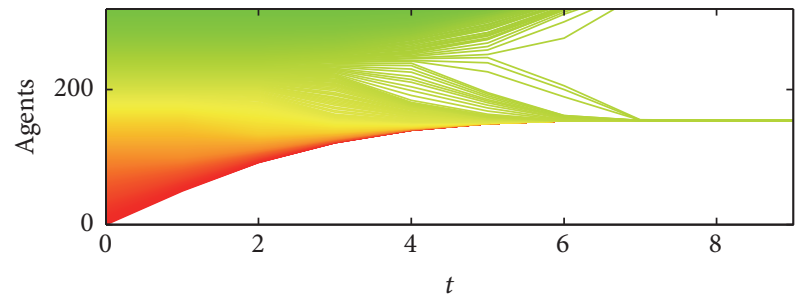

(f)

FIGURE 3: Opinion evolution in our constructed BA scale-free network: in each plot, the $x$-axis is time and the $y$-axis represents the nodes. (a) $\rightarrow$ (f) describe opinion evolution with different numbers of agents.

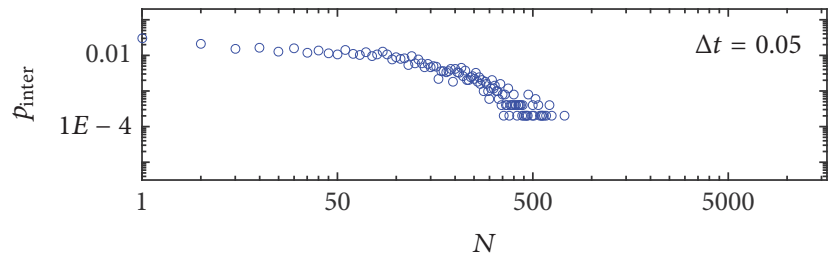

(a)

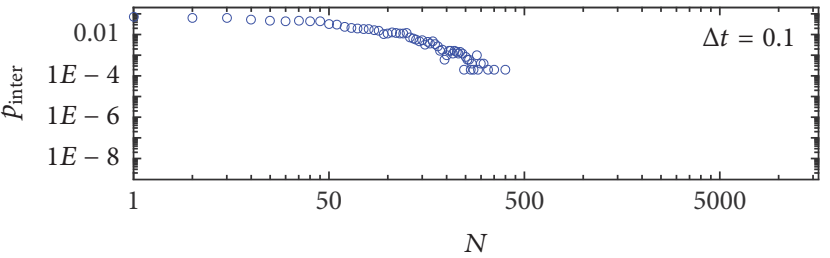

(b)

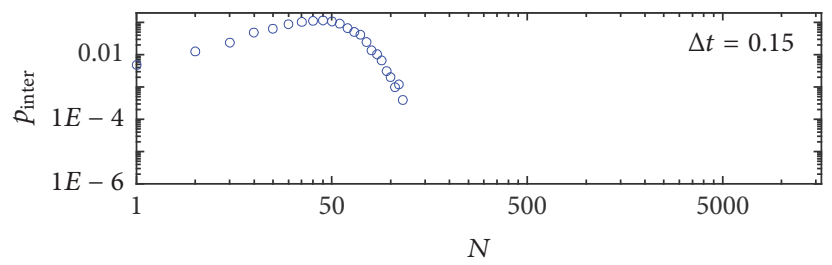

(c)

Figure 4: Histograms of cumulative group size in the consensus state for $\Delta t=0.05,0.1,0.15, \mu=0.4$, and the $x$-axis is $N_{i}$. The results are averaged on $10^{5}$ iterations of the system.

of China (14ATQ005), the Humanities and Social Sciences Research Youth Foundation of Ministry of Education of China (15YJCZH197), the Jiangxi Provincial Natural Science Foundation (20161BAB202055), the China Postdoctoral
Science Foundation (2015M580931), the Social Science Planning Projects in Jiangxi Province (14TQ07, 16TQ05), the Key Research Foundation of the Jiangxi Department of Education (GJJ150791), the Youth Fund of Humanities and Social 


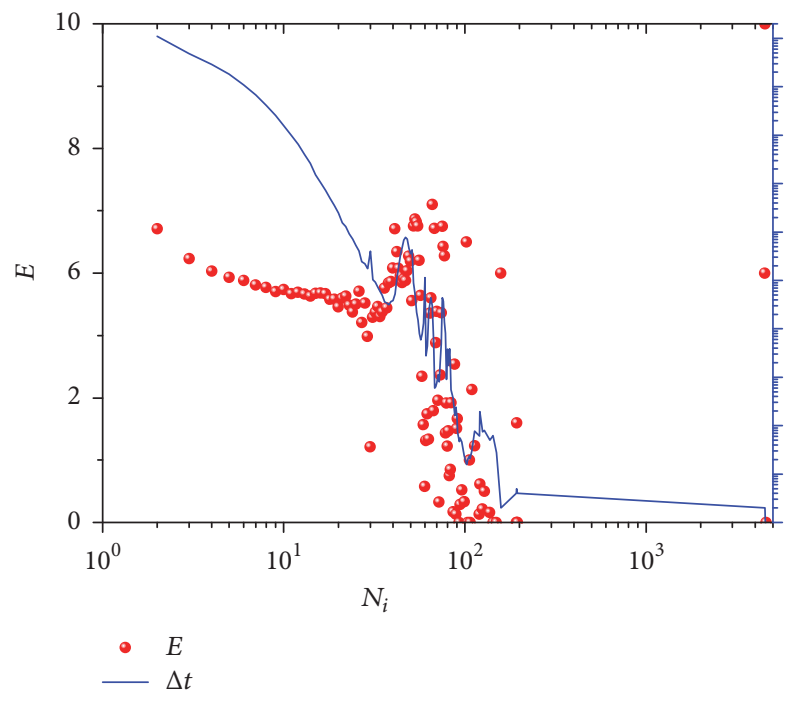

Figure 5: The change regulation of $E$ and $\Delta t$ at different $N_{i}$ (with log scale) in the system.

Sciences in Universities of Jiangxi Province (XW1505), and the Outstanding Youth Talent Support Program of JXSTNU (2015QNBJRC005).

\section{References}

[1] C. Castellano, S. Fortunato, and V. Loreto, "Statistical physics of social dynamics," Reviews of Modern Physics, vol. 81, no. 2, pp. 591-646, 2009.

[2] D. Stauffer, "Introduction to statistical physics outside physics," Physica A: Statistical Mechanics and Its Applications, vol. 336, no. 1-2, pp. 1-5, 2004.

[3] V. Sood and S. Redner, "Voter model on heterogeneous graphs," Physical Review Letters, vol. 94, no. 17, Article ID 178701, 2005.

[4] K. Suchecki, V. M. Eguíluz, and M. S. Miguel, "Voter model dynamics in complex networks: role of dimensionality, disorder, and degree distribution," Physical Review E, vol. 72, no. 3, Article ID 036132, 2005.

[5] A. Baronchelli, C. Castellano, and R. Pastor-Satorras, "Voter models on weighted networks," Physical Review E, vol. 83, no. 6, Article ID 066117, 2011.

[6] S. Galam, "Minority opinion spreading in random geometry," The European Physical Journal B, vol. 25, no. 4, pp. 403-406, 2002.

[7] P. L. Krapivsky and S. Redner, "Dynamics of majority rule in two-state interacting spin systems," Physical Review Letters, vol. 90, no. 23, Article ID 238701, 2003.

[8] S. Galam, "Contrarian deterministic effects on opinion dynamics: 'the hung elections scenario," Physica A: Statistical Mechanics and Its Applications, vol. 333, pp. 453-460, 2004.

[9] S. Galam, "The dynamics of minority opinions in democratic debate," Physica A: Statistical Mechanics and its Applications, vol. 336, no. 1-2, pp. 56-62, 2004.

[10] G. Deffuant, D. Neau, F. Amblard, and G. Weisbuch, "Mixing beliefs among interacting agents," Advances in Complex Systems, vol. 3, pp. 87-98, 2000.

[11] R. Hegselmann and U. Krause, "Opinion dynamics and bounded confidence, models, analysis and simulation," Journal of Artificial Societies and Social Simulation, vol. 81, no. 2, pp. 591646, 2009.

[12] A. C. R. Martins, C. D. B. Pereira, and R. Vicente, "An opinion dynamics model for the diffusion of innovations," Physica A: Statistical Mechanics and Its Applications, vol. 388, no. 15-16, pp. 3225-3232, 2009.

[13] K. Sznajd-Weron and J. Sznajd, "Opinion evolution in closed community," International Journal of Modern Physics C, vol. 11, no. 6, pp. 1157-1165, 2000.

[14] A. T. Bernardes, D. Stauffer, and J. Kertész, "Election results and the Sznajd model on Barabasi network," European Physical Journal B, vol. 25, no. 1, pp. 123-127, 2002.

[15] J. K. Shin, "Information accumulation system by inheritance and diffusion," Physica A: Statistical Mechanics and its Applications, vol. 388, no. 17, pp. 3593-3599, 2009.

[16] M. Afshar and M. Asadpour, "Opinion formation by informed agents," Journal of Artificial Societies and Social Simulation, vol. 13, no. 4, p. 5, 2010.

[17] C. Borghesi and S. Galam, "Chaotic, staggered, and polarized dynamics in opinion forming: the contrarian effect," Physical Review E, vol. 73, no. 6, Article ID 066118, 2006.

[18] L.-L. Jiang, D.-Y. Hua, and T. Chen, "Nonequilibrium phase transitions in a model with social influence of inflexible units," Journal of Physics A: Mathematical and Theoretical, vol. 40, no. 37, pp. 11271-11276, 2007.

[19] A.-L. Barabási and R. Albert, "Emergence of scaling in random networks," Science, vol. 286, no. 5439, pp. 509-512, 1999.

[20] D. J. Watts and S. H. Strogatz, "Collective dynamics of 'smallworld' networks," Nature, vol. 393, no. 6684, pp. 440-442, 1998.

[21] A. Szolnoki, G. Szabó, and M. Perc, "Phase diagrams for the spatial public goods game with pool punishment," Physical Review E, vol. 83, no. 3, Article ID 036101, 2011.

[22] G. Iñiguez, J. Kertész, K. K. Kaski, and R. A. Barrio, "Phase change in an opinion-dynamics model with separation of time scales," Physical Review E, vol. 83, no. 1, Article ID 016111, 2011.

[23] C. E. Shannon, "A mathematical theory of communication," Bell System Technical Journal, vol. 27, no. 3, pp. 379-423, 1948. 


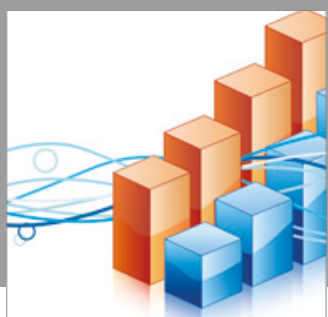

Advances in

Operations Research

vatersals

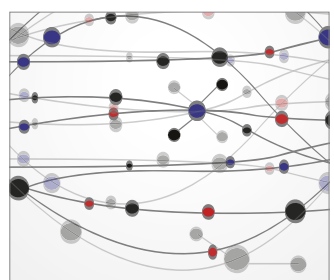

\section{The Scientific} World Journal
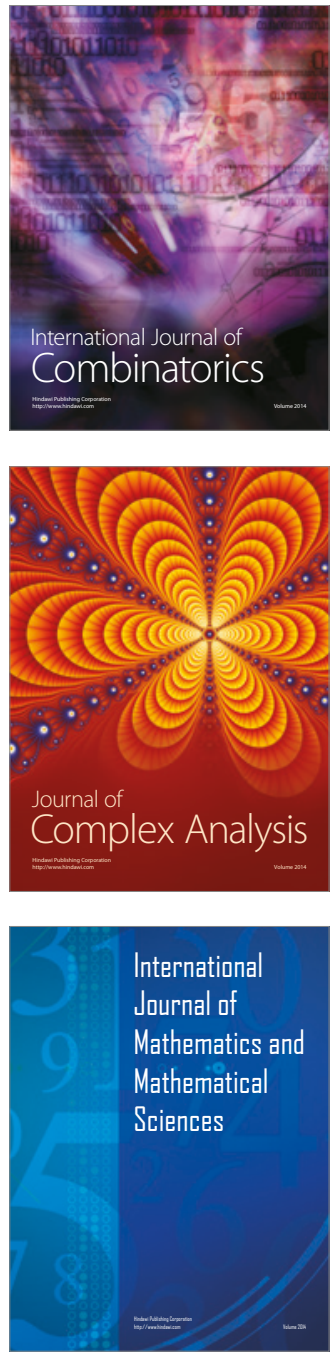
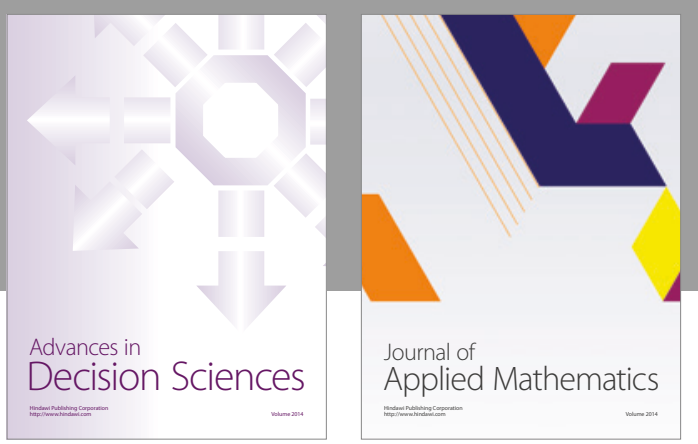

Algebra

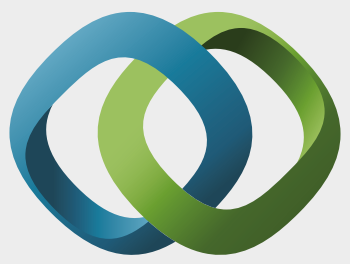

\section{Hindawi}

Submit your manuscripts at

https://www.hindawi.com
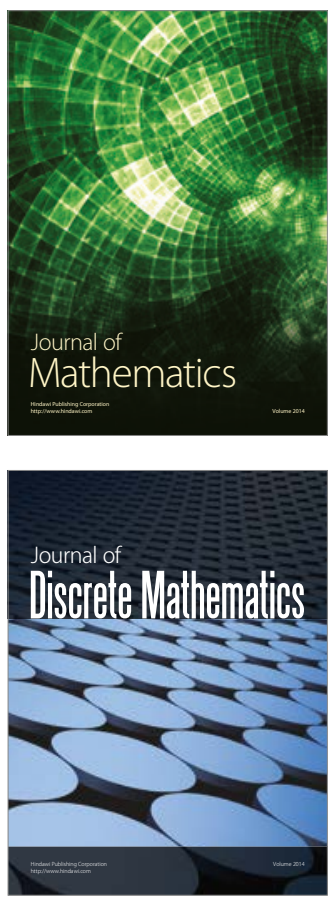

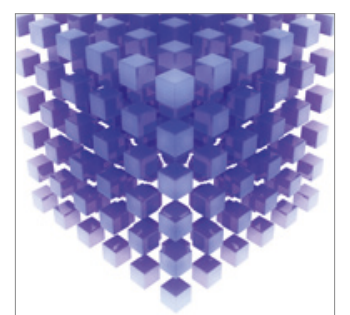

Mathematical Problems in Engineering
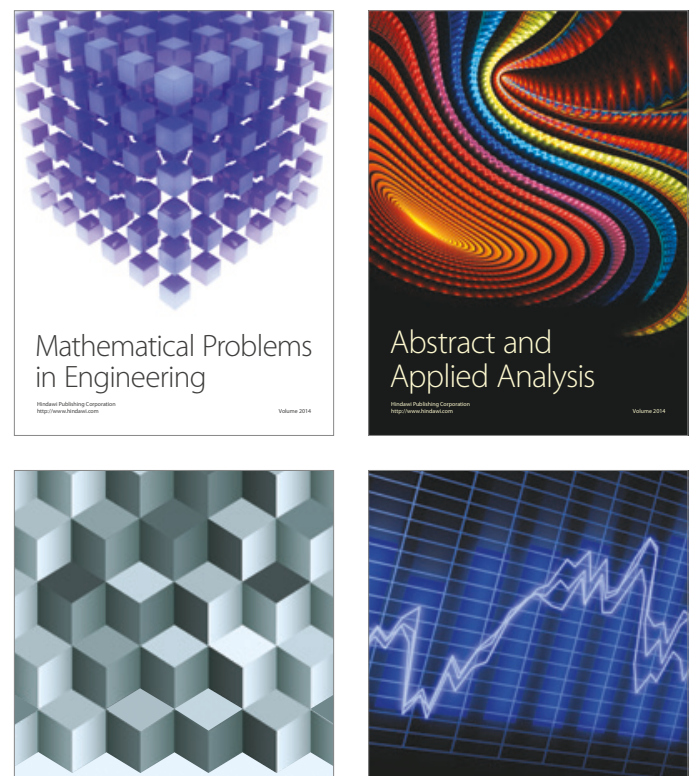

Journal of

Function Spaces

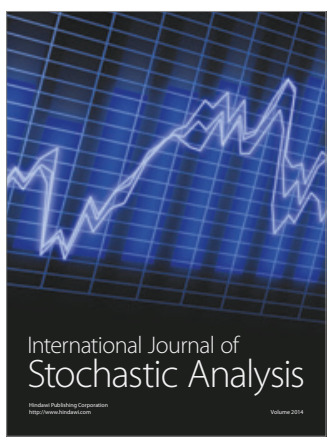

Probability and Statistics
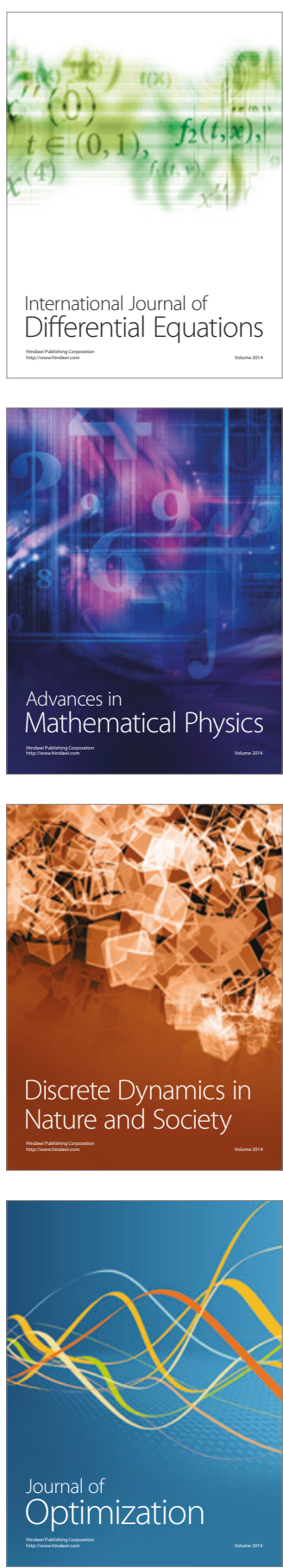